\title{
Influence of Different Dosage Forms on Pharmacokinetics of 6 Alkaloids in Raw Aconiti Kusnezoffii Radix (Caowu) and Chebulae Fructus- (Hezi-) Processed Caowu by UPLC-MS/MS
}

\author{
Meiru Zhi, Kaiyang Liu, Shu Han, Jinkai Xu, Weifei Li, Feng Li, Xitao Han, Yanan Tang, \\ Ziqin Liu, Hongyue Wang, and Hong Du
}

Beijing University of Chinese Medicine, Beijing 102488, China

Correspondence should be addressed to Hong Du; duhong@vip.163.com

Received 12 May 2020; Revised 6 September 2020; Accepted 12 September 2020; Published 22 September 2020

Academic Editor: Muhammad Hassham Hassan Bin Asad

Copyright (C) 2020 Meiru Zhi et al. This is an open access article distributed under the Creative Commons Attribution License, which permits unrestricted use, distribution, and reproduction in any medium, provided the original work is properly cited.

\begin{abstract}
Purpose. To study the pharmacokinetics of the 6 alkaloids (aconitine, mesaconitine, hypaconitine, benzoylaconine, benzoylmesaconine, and benzoylhypaconine) in raw Aconiti Kusnezoffii Radix (Caowu) (RC) and Chebulae Fructus- (Hezi-) processed Caowu (HC) in the rats being, respectively, administrated with RC and HC in the dosage forms of powder and decoction and to demonstrate the mechanism of detoxification of HC. Methods. The rats were randomly divided into 4 groups and, respectively, given RC powder, HC powder, RC decoction, and HC decoction by intragastric administration. The contents of the 6 alkaloids in the plasma of the rats were detected at different time points by the UPLC-MS/MS method, and DAS 3.2.7 software was used to calculate, compare, and analyze the detected pharmacokinetic parameters. Results. Compared with those of the RC powder, the values of $\mathrm{AUC}_{0-t}$ and $C_{\max }$ of the $\mathrm{HC}$ powder were all reduced, whereas the values of $t_{1 / 2 z}$ and $T_{\max }$ were mostly increased. Compared with those of the RC powder, the values of $\mathrm{AUC}_{0-t}, C_{\max }$, and $t_{1 / 2 z}$ of the $\mathrm{RC}$ decoction were decreased and the value of $T_{\max }$ of the RC decoction was increased. Compared with those of the RC decoction, the values of AU $\mathrm{C}_{0-t}, t_{1 / 2 z}$, and $C_{\max }$ of the diester diterpenoid alkaloids of the $\mathrm{HC}$ decoction were all increased. However, there was no marked difference between the pharmacokinetic parameters of the HC powder and the HC decoction. Conclusions. A decrease in the level of absorption and in the rate of elimination of the alkaloids can be detected when HC is administrated in the dosage form of the powder, explaining that in traditional Mongolian medicine (TMM), the purpose of using HC in the dosage forms of pills and powder is for decreasing the toxicity and prolonging the efficacy duration of HC. Decocting can greatly decrease the plasma concentration of the diester diterpenoid alkaloids in RC and increase their rate of elimination. The influence of decocting on RC is greater than that on $\mathrm{HC}$, explaining the rationality of the steaming and boiling methods for processing Caowu and the rationality of boiling Caowu for a longer time beforehand in preparing an herb decoction containing Caowu in TCM.
\end{abstract}

\section{Introduction}

Aconiti Kusnezoffii Radix, the root of plant Aconitum kusnezoffii Reichb, is named Caowu in traditional Chinese medicine (TCM) [1]. It can be used to treat paralysis and deal with various aches and pains, including arthralgia [2-4]. Caowu was first recorded in Sheng Nong's Herbal Classic and classified as a toxic herb in the book. In TCM clinical practice, although Caowu is mostly used externally, it can be used internally after being processed. The earliest recording of the processing methods for Caowu, such as peeling, boiling in water, and decocting with honey, can be found in Synopsis of Prescriptions of the Golden Chamber.

In modern times, Caowu is a commonly used herb in both TCM and traditional Mongolian medicine (TMM). In TCM, Caowu is usually processed by the boiling or steaming method, which can turn the highly toxic diester diterpenoid alkaloids hydrolyzed into less toxic monoester diterpenoid alkaloids and amine-diterpenoid alkaloids and enlarge the security window of Caowu. In TMM, Caowu is usually 
TABLE 1: The contents of the 6 alkaloids in different Caowu samples.

\begin{tabular}{|c|c|c|c|c|c|}
\hline Kinds of alkaloids & $\mathrm{RC}(\%)$ & $\mathrm{HC}(\%)$ & WSC (\%) & $\mathrm{RCD}(\%)$ & $\operatorname{HCD}(\%)$ \\
\hline $\mathrm{AC}$ & 0.363 & 0.384 & 0.227 & 0.060 & 0.105 \\
\hline MA & 0.493 & 0.302 & 0.328 & 0.000 & 0.025 \\
\hline HA & 0.019 & 0.007 & 0.010 & 0.000 & 0.001 \\
\hline BAC & 0.001 & 0.004 & 0.004 & 0.022 & 0.011 \\
\hline BMA & 0.086 & 0.100 & 0.069 & 0.410 & 0.247 \\
\hline BHA & 0.001 & 0.006 & 0.004 & 0.026 & 0.008 \\
\hline Total amount of DDA & 0.875 & 0.693 & 0.565 & 0.060 & 0.131 \\
\hline Total amount of MDA & 0.088 & 0.11 & 0.077 & 0.458 & 0.266 \\
\hline
\end{tabular}

Notes: DDA: diester diterpenoid alkaloids; MDA: monoester diterpenoid alkaloids.

processed with Chebulae Fructus, the fruits of Terminalia chebula Retz. and Terminalia chebula Retz, var. tomentella Kurt., which is called Hezi in TCM, but up to now, the mechanism of the detoxification of the Hezi-processing method remains unclear. Our previous study showed that the contents of diester diterpenoid alkaloids in Hezi-processed Caowu (HC) were slightly lower than those in raw Caowu (RC) but higher than those in water-soaked Caowu (WSC); although the contents of 3 diester diterpenoid alkaloids in both $\mathrm{RC}$ and $\mathrm{HC}$ decoctions were decreased, those in the HC decoction decreased at a lower level (Table 1), explaining that decocting could accelerate the hydrolysis of diester diterpenoid alkaloids whereas the Hezi-processing method could prevent the loss and hydrolysis of the 3 diester diterpenoid alkaloids to a certain degree. However, the acute toxicity of the $\mathrm{HC}$ powder was found to be significantly smaller than that of the RC powder although the contents of the 3 diester diterpenoid alkaloids in the RC powder were slightly higher than those in the HC powder [5-8]. Therefore, we speculated that the mechanism of detoxification of $\mathrm{HC}$ may be related to the in vivo absorption of the alkaloids.

Compared with the synthetic medicines, the chemical components of a single TCM herb or a group of herbs in a formula are usually complicated, and they can chemically interact with each other under certain conditions. In vitro, when an herb (or herbs in a formula) is being decocted or processed by a certain method, complicated chemical interactions of these components may occur, and in vivo, such interaction of the components will affect the absorption, distribution, and metabolism of these components [9]. In the present study, we plan to explore the mechanism of detoxification of the $\mathrm{HC}$ and the influence of different dosage forms on the toxicity of Caowu, by studying the pharmacokinetics of the 3 diester diterpenoid alkaloids, namely, aconitine (AC), mesaconitine (MA), and hypaconitine (HA), and the 3 monoester diterpenoid alkaloids, namely, benzoylaconine (BAC), benzoylmesaconine (BMA), and benzoylhypaconine (BHA), which are the 6 main bioactive components in Caowu [10-12] (Figure 1). As Caowu is mainly used in decoction in TCM and in powder and pills in TMM, RC powder (RCP), HC powder (HCP), RC decoction (RCD), and $\mathrm{HC}$ decoction (HCD) were chosen as the samples in our present study for detecting the pharmacokinetic parameters of the 6 alkaloids in Caowu.

\section{Experimental}

\subsection{Experimental Materials}

2.1.1. Standard Substances. The standard substances of the 6 alkaloids, AC (purity $>98.8 \%$ ), MA (purity $>99.2 \%$ ), HA (purity $>98.5 \%$ ), BAC (purity $>99.1 \%$ ), BMA (purity $>$ $98.6 \%$ ), and BHA (purity $>94 \%$ ), were all obtained from the National Institutes for Food and Drug Control (Beijing, China). The internal standard (I.S.) substance, reserpine (purity $>98 \%$ ), was purchased from the Shanghai Yuanye Bio-Technology Co., Ltd. (Shanghai, China).

2.1.2. Reagents and Solvents. The guaranteed reagents, methanol, acetonitrile, and formic acid, and some analytical reagents were all purchased from Thermo Fisher Scientific (Waltham, MA, USA). The ultrapure water was prepared by the Milli-Q ${ }^{\circledR}$ Reference Water Purification System (Merck Millipore, Darmstadt, Germany).

2.1.3. TCM Herbs. Aconiti Kusnezoffii Radix, namely, Caowu in TCM, was purchased from Beijing Huamiao Pharmaceutical Co., Ltd. (Beijing, China), and Chebulae Fructus, namely, Hezi in TCM, was purchased from Beijing Heyanling Pharmaceutical Co., Ltd. (Beijing, China), and both of them were authenticated by Prof. Jingjuan Wang from the School of Chinese Materia Medica of Beijing University of Chinese Medicine (BUCM). HC was prepared by one of our research team members.

2.2. Experimental Animals. Twenty-four male SpragueDawley (SD) rats were provided by SPF (Beijing) Biotechnology Co., Ltd. (Beijing, China). Weighing $200 \pm 20$ g, the rats were raised under the following conditions: humidity $=55$ $\pm 5 \%$, temperature $=22 \pm 2^{\circ} \mathrm{C}$, and light $=12 / 12 \mathrm{~h}$. All the rats had free access to water and foodstuff and were acclimated for at least 3 days before being used in an experiment. Before attending an experiment, all the rats were fasted for $12 \mathrm{~h}$, during which, they were allowed to drink water freely. All animal experiments were made according to the Regulations of Beijing Municipality on the Administration of 


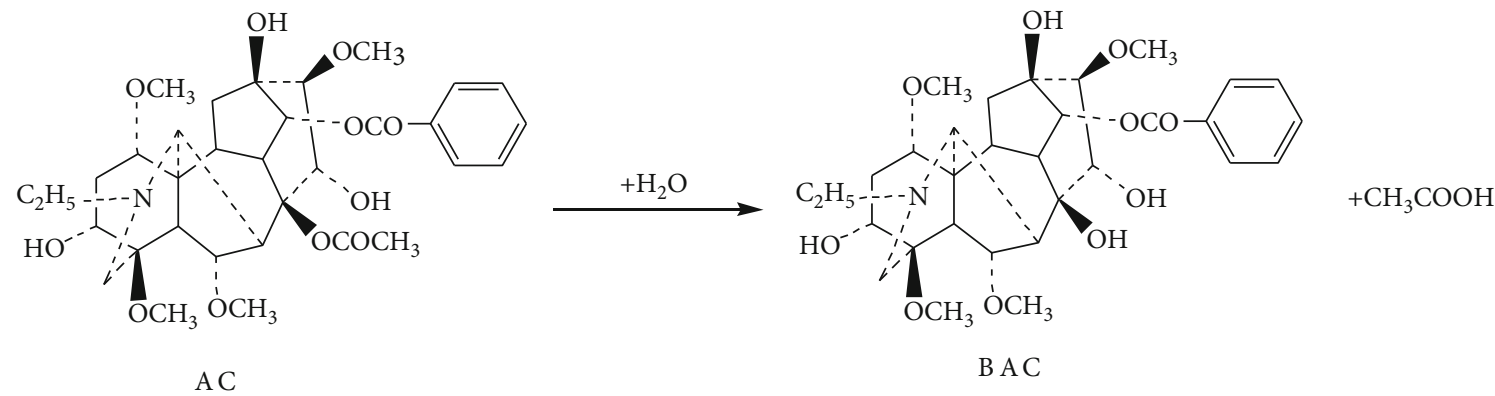

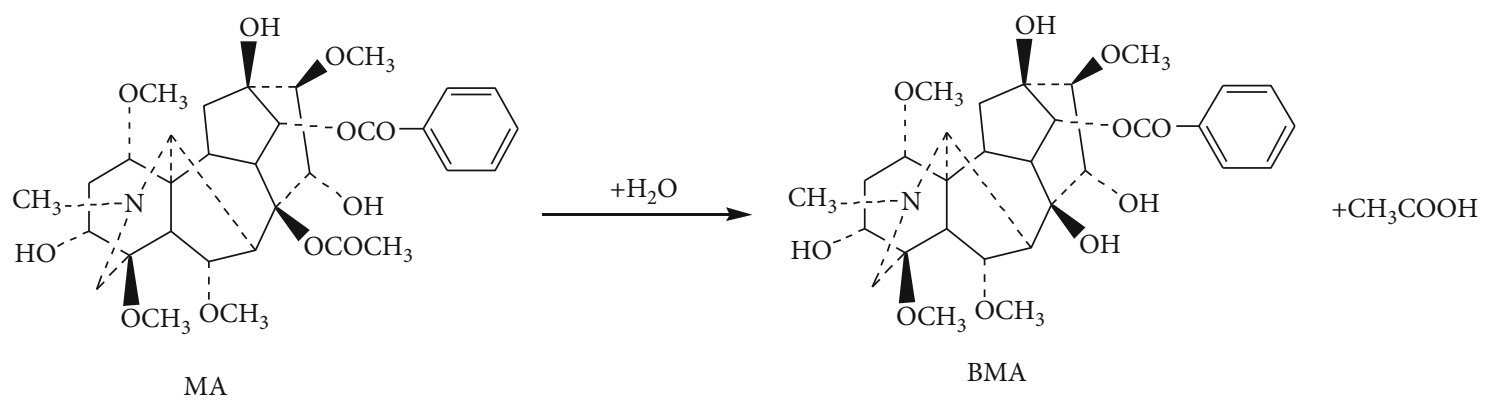

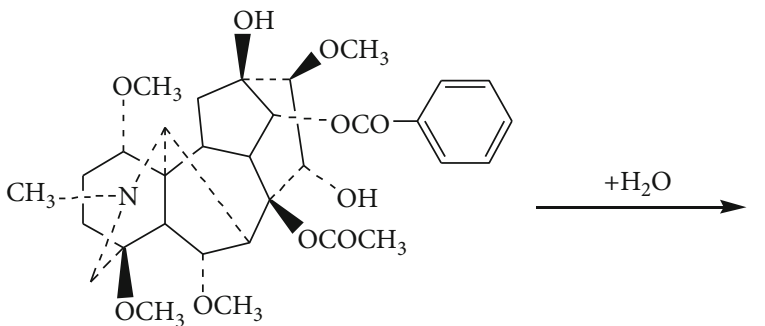

HA

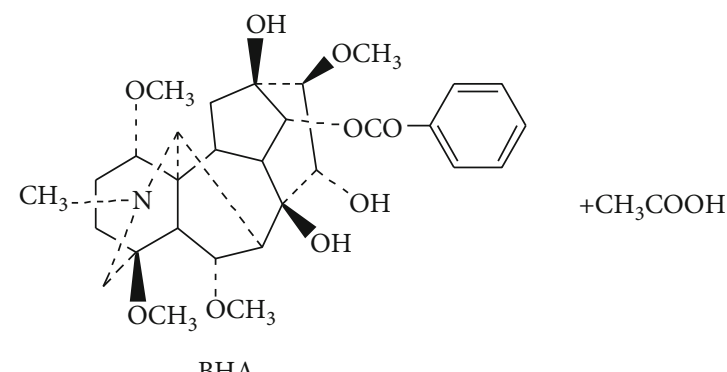

Figure 1: The reactions of diester diterpenoid alkaloids being hydrolyzed to monoester diterpenoid alkaloids. Notes: AC: aconitine; MA: mesaconitine; HA: hypaconitine; BAC: benzoylaconine; BMA: benzoylmesaconine; BHA: benzoylhypaconine.

Laboratory Animals and were authorized by the BUCM Animal Ethics Committee.

\subsection{Experimental Methods}

2.3.1. UPLC-MS/MS System and the Conditions for Operation. A Waters Xevo TQ-S Triple Quad Mass Spectrometer was connected to an ACQUITY ${ }^{\mathrm{TM}}$ UPLC system (Waters Corp., Milford, MA, USA) via an electrospray ionization source (ESI) interface. Gradient elution was performed on a Waters ACQUITY ${ }^{\mathrm{TM}}$ UPLC $\mathrm{BEH} \mathrm{C}_{18}$ column $(100 \mathrm{~mm} \times 2.1 \mathrm{~mm}, 1.7 \mu \mathrm{m})$ at $40^{\circ} \mathrm{C}$. The mobile phase was comprised of acetonitrile and $0.1 \%$ formic acid water, and the flow rate was $0.3 \mathrm{~mL} / \mathrm{min}$. The changes in the gradient were as follows: acetonitrile was kept at $5 \%$ for $0.5 \mathrm{~min}$, acetonitrile was increased from $5 \%$ to $45 \%$ for $0.5-1.5 \mathrm{~min}$, acetonitrile was raised to $47 \%$ from $45 \%$ for $1.5-4.5 \mathrm{~min}$, acetonitrile was increased to $95 \%$ from $47 \%$ for $4.5-6.5 \mathrm{~min}$ and was maintained this level for $1 \mathrm{~min}$, and acetonitrile was decreased to $5 \%$ for $7.5-9.5 \mathrm{~min}$ and was maintained at this level for $0.5 \mathrm{~min}$. The injection volume was $2 \mu \mathrm{L}$.
The quantitative detection of the alkaloids was operated via multiple-reaction monitoring (MRM) in a positive ion mode. The working parameters of mass spectrometry were as follows: the capillary voltage was $0.58 \mathrm{kV}$, the desolvation gas (nitrogen) flow was $993 \mathrm{~L} / \mathrm{h}$, the cone gas (nitrogen) flow was $1 \mathrm{~L} / \mathrm{h}$, the source temperature was $149^{\circ} \mathrm{C}$, and the desolvation temperature was $498^{\circ} \mathrm{C}$. All of the data were collected and processed by using the MassLynx ${ }^{\mathrm{TM}}$ NT 4.2 software (Waters Corp., Milford, MA, USA).

2.3.2. Preparation of Standard Substance and Quality Control Working Solutions. A series of standard substance and I.S. ( $500 \mathrm{ng} / \mathrm{mL}$ ) working solutions was prepared with methanol. The standard substance working solutions were prepared according to the method described in the Section 2.3.3 of the present paper, except for the methanol $(10 \mu \mathrm{L})$ which was substituted for the standard substance working solution $(10 \mu \mathrm{L})$, and the final concentrations of the standard substance working solutions in the series were $0.05,0.1,0.5,1$, $5,10,50,100,250$, and $500 \mathrm{ng} / \mathrm{mL}$. The quality control 
working solutions of various concentrations (0.1, 50, and $400 \mathrm{ng} / \mathrm{mL}$ ) were also prepared according to the same method. All the prepared working solutions were kept in a refrigerator at $4^{\circ} \mathrm{C}$ before use.

2.3.3. Preparation of the Plasma Sample. $10 \mu \mathrm{L}$ of methanol, $10 \mu \mathrm{L}$ of work solution of I.S. ( $500 \mathrm{ng} / \mathrm{mL}), 400 \mu \mathrm{L}$ of acetonitrile, and $100 \mu \mathrm{L}$ of plasma sample were mixed and vortexed for $1 \mathrm{~min}$ in a $2 \mathrm{~mL}$ centrifuge tube and then centrifuged at $10,000 \mathrm{rpm}$ for $15 \mathrm{~min}$. The supernatant was then transferred into another $2 \mathrm{~mL}$ centrifuge tube and dried under nitrogen. The residue was redissolved in $100 \mu \mathrm{L}$ of methanol.

\subsubsection{Validation of the Experimental Methods}

(1) Selectivity. The blank rat plasma samples, blank rat plasma samples spiked with the 6 standard substances and I.S., and the rat plasma samples after intragastric administration of RCP, HCP, RCD, and HCD for $1 \mathrm{~h}$ were used for evaluating selectivity.

(2) Linearity. In the calibration curve, $X$ was established as the analyte concentration and $Y$ as the ratio of the analyte peak area to the I.S. peak area, in the concentration range of $0.05-500 \mathrm{ng} / \mathrm{mL}$.

(3) Precision and Accuracy. The intra- and interday precision and accuracy were detected by measuring the 6 repetitive quality control samples in 1 day for 3 successive days. Precision was expressed as the relative standard deviation (R.S.D.), and accuracy was expressed as the proportion of the mean measured concentration to the added standard concentration.

(4) Recovery and Matrix Effect. Peak A was obtained by adding standard substance working solutions of 6 alkaloids of 3 concentrations $(0.1,50$, and $400 \mathrm{ng} / \mathrm{mL})$ to the nonprocessed blank plasma. Peak B was achieved by adding standard substance working solutions to the protein-removed blank plasma. Peak $\mathrm{C}$ was achieved by only using the standard substance working solutions of 3 concentrations. Peaks A, B, and $\mathrm{C}$ were adjusted by I.S. and expressed as the ratio of the peak area of 6 alkaloids to the peak area of I.S. Recovery was expressed as the ratio of peak $A$ to peak $B$, and matrix effect was expressed as the ratio of peak B to peak C.

(5) Stability. Stability of all the samples for the experiments in our present study were detected after they were stored in a refrigerator at $4^{\circ} \mathrm{C}$ for $12 \mathrm{~h}$ and were represented as the percentage of the measured concentration with the added substance concentration.

\subsubsection{Preparation of $R C$ and HC Powder and Decoction}

(1) Preparation of HC. Hezi was crushed into uniform pieces and soaked in water for $1 \mathrm{~h}$ and then decocted in water for $1 \mathrm{~h}$ to gain the Hezi decoction. Afterwards, Caowu was soaked in the Hezi decoction in a ratio of 2:1 (Caowu: Hezi) for 3 days to gain $\mathrm{HC}[5]$.
TABLE 2: Mass spectrometry selection channel and related parameters.

\begin{tabular}{lcccc}
\hline $\begin{array}{l}\text { Detecting } \\
\text { items }\end{array}$ & Precursor ion & Product ion & Cone volt. & Col. energy \\
\hline AC & 646.3 & 586.3 & 14.0 & 32.0 \\
MA & 632.3 & 572.4 & 14.0 & 30.0 \\
HA & 616.4 & 105.0 & 18.0 & 58.0 \\
BAC & 604.4 & 105.0 & 20.0 & 56.0 \\
BMA & 590.3 & 105.0 & 52.0 & 62.0 \\
BHA & 574.3 & 105.0 & 58.0 & 62.0 \\
I.S. & 609.5 & 195.1 & 86.0 & 64.0 \\
\hline
\end{tabular}

(2) The Preparation of RC and HC Powder and Decoction. Preparation of $\mathrm{RC}$ or HC powder suspension: $\mathrm{RC}$ or $\mathrm{HC}$ was ground into powder and then mixed with $0.5 \%$ sodium carboxymethyl cellulose solution to form a suspension, the concentration of which was $0.1 \mathrm{~g} / \mathrm{mL}$. The RC or HC powder suspension should be prepared just before the intragastric administration.

Preparation of RC or HC decoction: RC or HC was soaked in water for 30 min after being sliced. After soaking, the RC or $\mathrm{HC}$ slices were decocted in water in a ratio of $1: 10(\mathrm{RC}$ or $\mathrm{HC}$ : water, $w: v)$ for $30 \mathrm{~min}$ for 2 times. The filtrates of the 2 times of decocting were merged and condensed by vacuum concentration at $40^{\circ} \mathrm{C}$ for gaining a decoction at a concentration of $0.1 \mathrm{~g} / \mathrm{mL}$. The RC or HC decoction should be prepared just before the intragastric administration.

2.3.6. Pharmacokinetic Detection. Twenty-four rats were randomly and evenly divided into 4 groups, i.e., groups of RCP, HCP, RCD, and RCD. According to the Pharmacopoeia of the People's Republic of China, the dosage of boilingprocessed Caowu is 1.5-3 g/day [1]. By a human-rat conversion on the basis of the body surface area, the dosage of the boiling-processed Caowu for rats is found to be 0.135 $0.27 \mathrm{~g} / \mathrm{kg} /$ day. However, the results of our previous study proved that this dosage was too small to be used in the experiments in our present study. After doing preliminary experiments repeatedly, we finally decided to adopt the dosage of $1 \mathrm{~g} / \mathrm{kg} /$ day for the rats in the 4 groups. The plasma samples (about $0.5 \mathrm{~mL}$ ) were collected at the time points of $0.25,0.5$, $1,1.5,2,3$, and $4 \mathrm{~h}$, and then, they were put into the heparinized centrifuge tubes for centrifuging at 4,000 rpm for $15 \mathrm{~min}$, and the supernatants were stored in a refrigerator at $-20^{\circ} \mathrm{C}$ before being used for analysis.

The mean plasma concentration-time curves were plotted by Origin Software (version 9.0). The pharmacokinetic parameters were calculated in a noncompartmental model via DAS (version 3.2.7, Mathematical Pharmacology Professional Committee of China), which was determined by the application of Akaike's Information Criterion (AIC). For each rat, the maximum plasma concentration $\left(C_{\max }\right)$ and its corresponding time $\left(T_{\max }\right)$ were determined by visual inspection of the profiles. The apparent terminal elimination rate constant $(\lambda)$ was calculated by linear regression of the natural logarithms of the terminal plasma concentrations. The terminal half-life $\left(t_{1 / 2 z}\right)$ was derived from $0.693 / \lambda$. The area under 

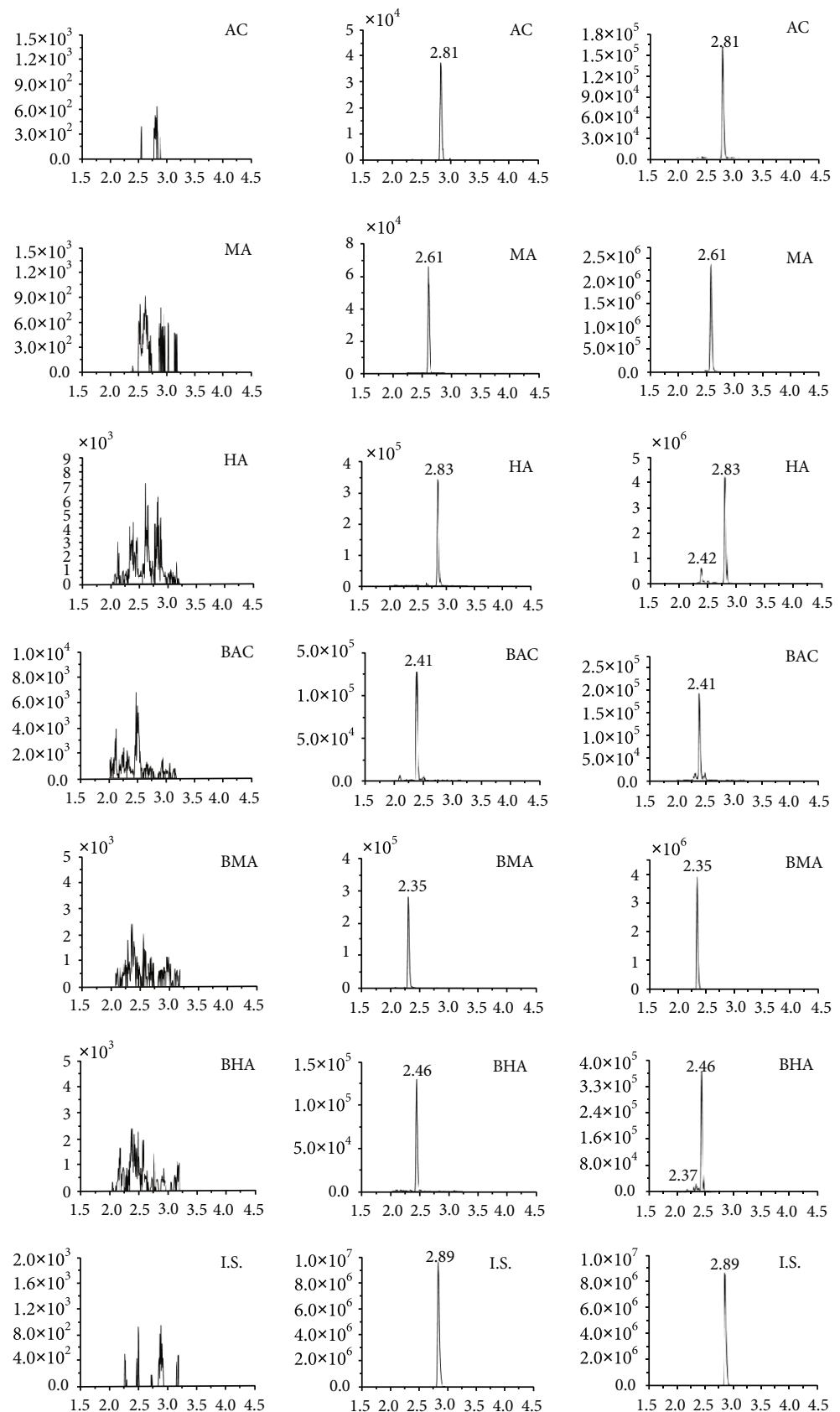

(a)

(b)

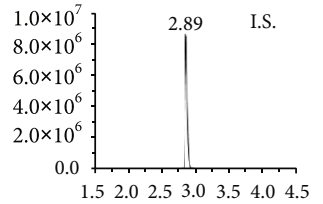

(c)

Figure 2: Continued. 

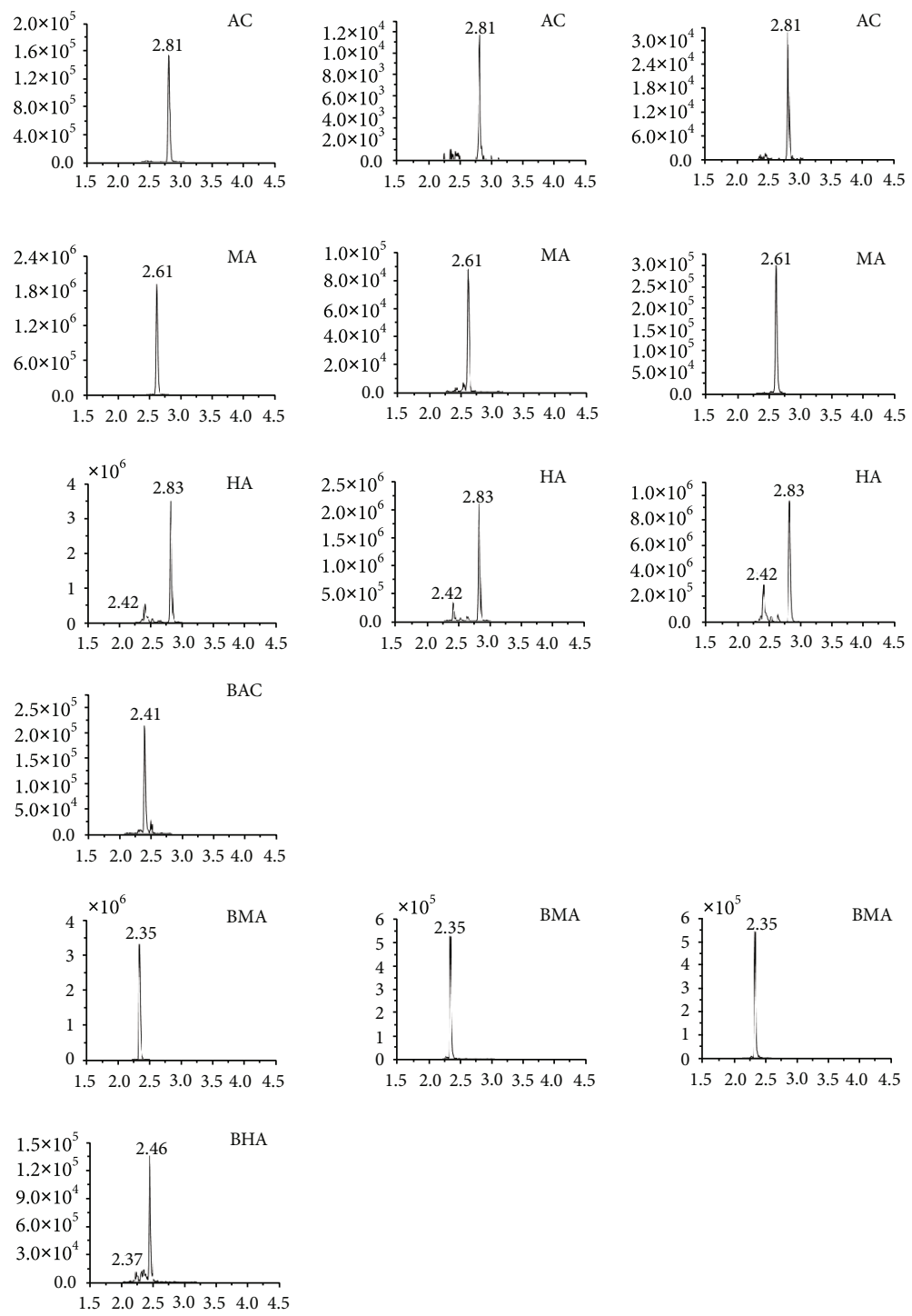

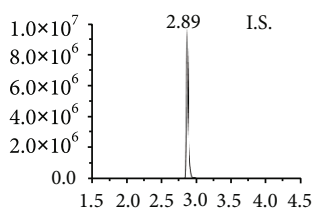

(d)

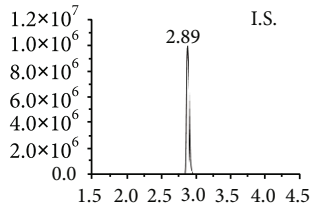

(e)

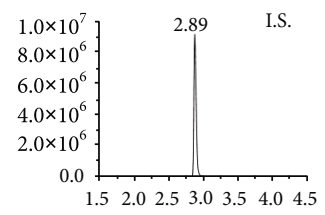

(f)

FIGURE 2: MRM chromatograms of the 6 alkaloids and I.S.: (a) blank plasma; (b) blank plasma mixed with the 6 alkaloids and I.S.; (c-f) $1 \mathrm{~h}$ plasma sample after administration of RCP (c), HCP (d), RCD (e), and HCD (f).

TABLE 3: Regression data for the 6 alkaloids.

\begin{tabular}{lccc}
\hline Alkaloids & Calibration & $R^{2}$ & Linear range (ng/mL) \\
\hline AC & $Y=0.0879452 X+0.0532361$ & 0.996 & $0.05 \sim 500$ \\
MA & $Y=0.119817 X+0.0288623$ & 0.992 & $0.05 \sim 500$ \\
HA & $Y=0.10836 X+0.223721$ & 0.994 & $0.05 \sim 500$ \\
BAC & $Y=0.0777178 X+0.059877$ & 0.993 & $0.05 \sim 500$ \\
BMA & $Y=0.0663272 X+0.580478$ & 0.991 & $0.05 \sim 500$ \\
BHA & $Y=0.0402479 X+0.116155$ & 0.991 & $0.05 \sim 500$ \\
\hline
\end{tabular}


TABLe 4: Precision and accuracy of the 6 alkaloids (intraday: $n=6$; interday: 3 days).

\begin{tabular}{|c|c|c|c|c|c|}
\hline \multirow{2}{*}{ Alkaloids } & \multirow{2}{*}{ Concentration $(\mathrm{ng} / \mathrm{mL})$} & \multicolumn{2}{|c|}{ Intraday } & \multicolumn{2}{|c|}{ Interday } \\
\hline & & Accuracy (\%) & Precision (R.S.D., \%) & Accuracy (\%) & Precision (R.S.D., \%) \\
\hline \multirow{3}{*}{$\mathrm{AC}$} & 0.1 & 94.00 & 12.78 & 95.00 & 4.10 \\
\hline & 50 & 91.04 & 4.51 & 91.17 & 1.41 \\
\hline & 400 & 93.79 & 6.34 & 95.20 & 1.30 \\
\hline \multirow{3}{*}{ MA } & 0.1 & 99.00 & 10.2 & 97.00 & 1.67 \\
\hline & 50 & 90.75 & 5.67 & 89.60 & 1.74 \\
\hline & 400 & 89.82 & 5.47 & 93.61 & 3.65 \\
\hline \multirow{3}{*}{ HA } & 0.1 & 98.00 & 11.12 & 102.00 & 4.84 \\
\hline & 50 & 91.55 & 5.78 & 93.18 & 2.54 \\
\hline & 400 & 89.45 & 4.60 & 92.91 & 4.07 \\
\hline \multirow{3}{*}{$\mathrm{BAC}$} & 0.1 & 90.00 & 14.23 & 93.00 & 5.30 \\
\hline & 50 & 95.39 & 6.56 & 94.42 & 0.90 \\
\hline & 400 & 98.29 & 7.49 & 97.30 & 2.42 \\
\hline \multirow{3}{*}{ BMA } & 0.1 & 103.00 & 11.89 & 96.00 & 7.93 \\
\hline & 50 & 96.51 & 7.93 & 93.01 & 6.80 \\
\hline & 400 & 98.06 & 6.60 & 99.68 & 1.70 \\
\hline \multirow{3}{*}{ BHA } & 0.1 & 104.00 & 11.92 & 95.00 & 8.98 \\
\hline & 50 & 93.24 & 10.14 & 95.02 & 1.74 \\
\hline & 400 & 95.22 & 5.39 & 97.24 & 1.81 \\
\hline
\end{tabular}

the curve to the last measured point $\left(\mathrm{AUC}_{0-t}\right)$ was calculated using the trapezoidal rule. The data were shown as mean \pm standard deviation (S.D.). The comparisons among the 4 groups were carried out by unpaired Student's $t$-test.

\section{Results}

3.1. Results of Validation of the Experimental Methods. In our present study, the MS/MS parameters were optimized in order to obtain the highest response. Table 2 showed the optimized mass transition ion pairs for quantification in the MRM mode, including precursor and product ions.

Acetonitrile, methanol, water, and formic acid, as the mobile phases, were evaluated in order to obtain their retention time and the appropriate response. It was found that compared with pure water, the $0.1 \%$ formic acid-containing water could obtain a better intensity and enhance the efficiency of ionization. Phenacetin, lappaconite hydrobromide, and reserpine, as the I.S., were evaluated, and finally, reserpine was chosen because it was found that its physicochemical properties and peak location were similar to the 6 alkaloids. The retention times of AC, MA, HA, BAC, BMC, $\mathrm{BHC}$, and I.S. were 2.81, 2.61, 2.83, 2.41, 2.35, 2.46, and $2.89 \mathrm{~min}$, respectively (Figure 2 ).

In order to remove the endogenous interference and obtain satisfactory results, different protein-removing reagents, such as methanol, acetonitrile, ethyl acetate, and diethyl ether, were tested, and finally, it was found that acetonitrile was the desired reagent.

The chromatograms in Figure 2 showed that there were no endogenous impurities interfering with the detection of the analytes and I.S. and that the specificity of the methods for detecting the analytes and I.S. could thereby be confirmed. The results of the calibration of the plasma samples are shown in Table 3, and it could be seen that the correlation coefficients $\left(R^{2}\right)$ for the 6 alkaloids were $\geq 0.99$. The R.S.D. of the intra- and interday precision for all the 6 alkaloids were less than $15 \%$, and the intra- and interday accuracies for all the 6 alkaloids were in a range of $89.45 \%$ to $104.00 \%$ (Table 4). The data of peaks A, B, and C are shown in Table 5, and it could be seen that the recoveries of the 6 alkaloids were above $82.04 \%$, and the range of the matrix effects was from $85.00 \%$ to $100.70 \%$, verifying that the methods used for the detection were feasible. The stability of the 6 alkaloids was found to be variable within $\pm 15 \%$ (Table 6 ).

3.2. Results of Pharmacokinetic Detection. The mean plasma concentration-time curves of the 6 alkaloids are shown in Figure 3, and the pharmacokinetic parameters detected after intragastric administration of RCP, HCP, RCD, and HCD are shown in Table 7. In the RCD and HCD groups, only the mean plasma concentrations and pharmacokinetic parameters of the alkaloids of AC, MA, HA, and BMA were calculated and compared, for the concentrations of the alkaloids $\mathrm{BAC}$ and BHA were too low to be detected.

The results of the detection showed that the mean plasma concentrations of the 6 alkaloids in HCP were significantly lower than those in RCP, and the results also showed that $\mathrm{AUC}_{0-t}$ and $C_{\max }$ of HCP were lower than those of RCP, whereas $T_{\max }$ and $t_{1 / 2 z}$ of HCP were higher than those of RCP, explaining that HCP could reduce the level and rate of absorption of the 6 alkaloids, delay the rate of elimination, and prolong the time of action. 
TABle 5: The data of peaks A, B, and C.

\begin{tabular}{|c|c|c|c|c|}
\hline Compound & Concentration $(\mathrm{ng} / \mathrm{mL})$ & $\mathrm{A}$ & $\mathrm{B}$ & $\mathrm{C}$ \\
\hline \multirow{3}{*}{$\mathrm{AC}$} & 0.1 & $0.061 \pm 0.001$ & $0.063 \pm 0.001$ & $0.065 \pm 0.003$ \\
\hline & 50 & $4.122 \pm 0.169$ & $4.957 \pm 0.181$ & $5.845 \pm 0.386$ \\
\hline & 400 & $33.734 \pm 1.847$ & $35.279 \pm 1.727$ & $38.799 \pm 4.024$ \\
\hline \multirow{3}{*}{ MA } & 0.1 & $0.041 \pm 0.001$ & $0.042 \pm 0.002$ & $0.043 \pm 0.003$ \\
\hline & 50 & $5.465 \pm 0.308$ & $6.316 \pm 0.393$ & $6.986 \pm 0.871$ \\
\hline & 400 & $43.075 \pm 2.356$ & $52.626 \pm 2.185$ & $59.124 \pm 5.443$ \\
\hline \multirow{3}{*}{ HA } & 0.1 & $0.234 \pm 0.001$ & $0.237 \pm 0.001$ & $0.239 \pm 0.003$ \\
\hline & 50 & $5.184 \pm 0.287$ & $6.0445 \pm 0.385$ & $7.046 \pm 1.194$ \\
\hline & 400 & $38.996 \pm 1.785$ & $47.690 \pm 3.695$ & $56.177 \pm 10.792$ \\
\hline \multirow{3}{*}{ BAC } & 0.1 & $0.068 \pm 0.001$ & $0.068 \pm 0.001$ & $0.069 \pm 0.002$ \\
\hline & 50 & $3.704 \pm 0.257$ & $3.829 \pm 0.245$ & $4.176 \pm 0.537$ \\
\hline & 400 & $30.836 \pm 1.972$ & $31.311 \pm 2.148$ & $33.730 \pm 4.377$ \\
\hline \multirow{3}{*}{ BMA } & 0.1 & $0.587 \pm 0.001$ & $0.587 \pm 0.001$ & $0.590 \pm 0.002$ \\
\hline & 50 & $3.423 \pm 0.233$ & $3.946 \pm 0.212$ & $4.271 \pm 0.572$ \\
\hline & 400 & $26.987 \pm 1.55$ & $28.085 \pm 1.449$ & $29.574 \pm 2.380$ \\
\hline \multirow{3}{*}{ BHA } & 0.1 & $0.120 \pm 0.001$ & $0.120 \pm 0.000$ & $0.119 \pm 0.001$ \\
\hline & 50 & $1.993 \pm 0.190$ & $2.146 \pm 0.144$ & $2.166 \pm 0.259$ \\
\hline & 400 & $15.445 \pm 0.825$ & $16.150 \pm 0.402$ & $16.441 \pm 0.525$ \\
\hline
\end{tabular}

TABLE 6: Recoveries, matrix effect, and stability of the 6 alkaloids $(n=6)$.

\begin{tabular}{|c|c|c|c|c|c|c|c|}
\hline Alkaloids & Concentration $(\mathrm{ng} / \mathrm{mL})$ & Extraction recovery (\%) & R.S.D. (\%) & Matrix effect (\%) & R.S.D. (\%) & $\begin{array}{c}\text { Stability } \\
\left(12 \mathrm{~h} \text { at } 4^{\circ} \mathrm{C}, \%\right)\end{array}$ & R.S.D. (\%) \\
\hline \multirow{3}{*}{$\mathrm{AC}$} & 0.1 & 97.11 & 1.57 & 97.65 & 2.64 & 95.83 & 14.42 \\
\hline & 50 & 83.31 & 7.22 & 85.00 & 4.87 & 93.49 & 4.40 \\
\hline & 400 & 95.75 & 6.30 & 91.42 & 6.54 & 88.65 & 7.54 \\
\hline \multirow{3}{*}{ MA } & 0.1 & 97.76 & 5.63 & 96.91 & 4.99 & 97.17 & 8.93 \\
\hline & 50 & 86.77 & 7.48 & 89.80 & 9.13 & 88.66 & 5.70 \\
\hline & 400 & 82.04 & 8.27 & 89.36 & 5.37 & 95.54 & 6.49 \\
\hline \multirow{3}{*}{ HA } & 0.1 & 99.09 & 0.63 & 98.94 & 0.75 & 96.33 & 14.06 \\
\hline & 50 & 86.07 & 8.92 & 87.24 & 11.96 & 91.58 & 9.56 \\
\hline & 400 & 82.20 & 9.43 & 86.40 & 11.18 & 96.15 & 7.48 \\
\hline \multirow{3}{*}{ BAC } & 0.1 & 99.54 & 2.58 & 98.54 & 2.06 & 89.83 & 10.89 \\
\hline & 50 & 97.20 & 10.67 & 92.49 & 8.79 & 94.91 & 10.03 \\
\hline & 400 & 98.79 & 8.00 & 93.48 & 6.76 & 94.14 & 4.31 \\
\hline \multirow{3}{*}{ BMA } & 0.1 & 99.77 & 0.18 & 99.80 & 0.20 & 100.50 & 11.24 \\
\hline & 50 & 87.16 & 11.59 & 93.33 & 9.46 & 97.10 & 8.89 \\
\hline & 400 & 96.46 & 10.05 & 95.21 & 5.20 & 98.80 & 6.86 \\
\hline \multirow{3}{*}{ BHA } & 0.1 & 100.42 & 0.70 & 100.70 & 0.63 & 108.00 & 12.17 \\
\hline & 50 & 96.35 & 12.10 & 99.74 & 7.09 & 98.07 & 9.28 \\
\hline & 400 & 98.74 & 3.44 & 98.25 & 1.65 & 97.91 & 1.71 \\
\hline
\end{tabular}



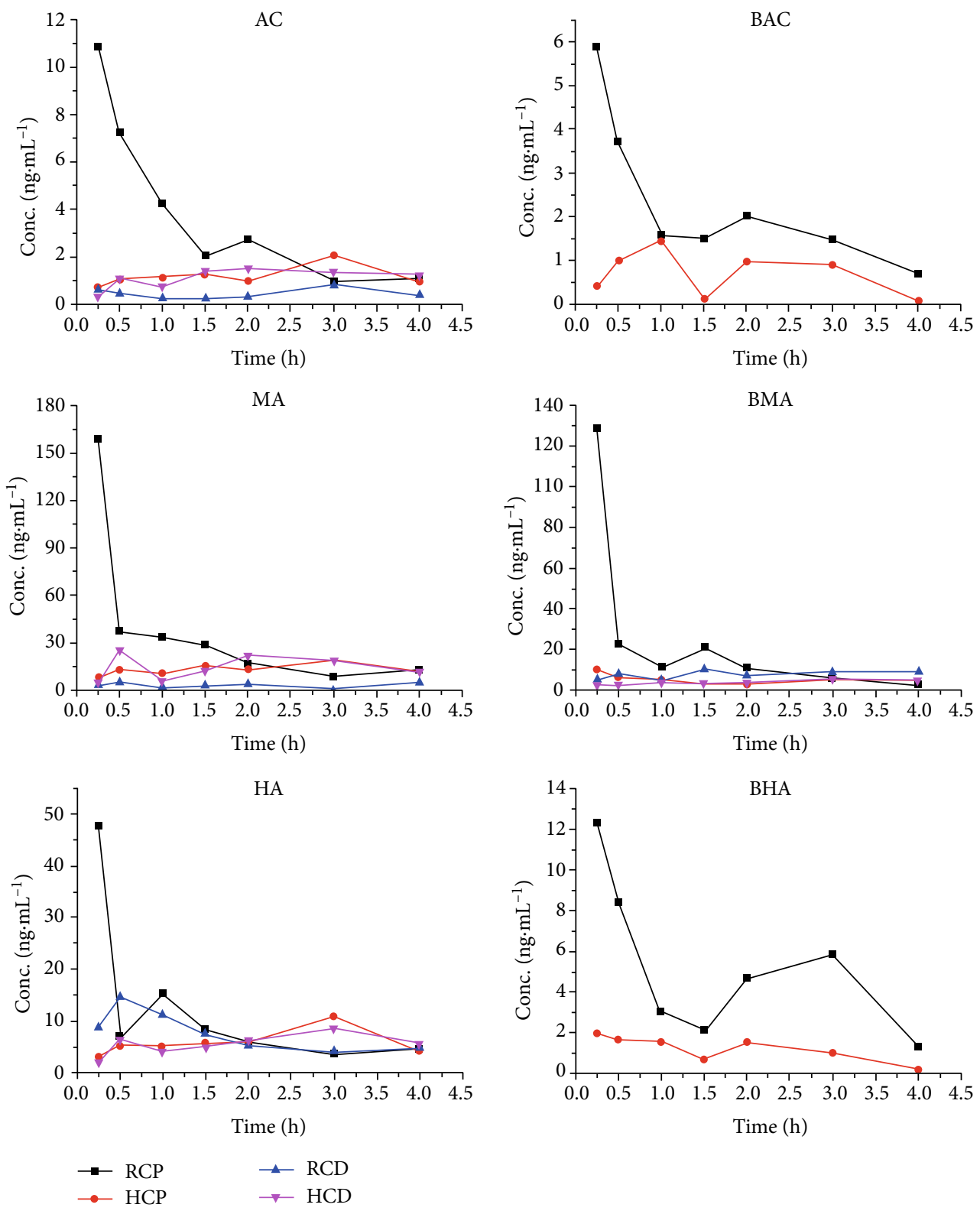

Figure 3: Mean plasma concentration-time curves.

The results of the detection also showed that compared with those in RCP, the mean plasma concentrations of AC and MA were markedly lower in RCD, with a significant difference concerning $\mathrm{AUC}_{0-t}, C_{\max }$, and $t_{1 / 2 z}$, implying that the level of the 2 diester diterpenoid alkaloids in plasma could be reduced and that their rate of elimination could be increased when RC was used in the dosage form of decoction.

At the same time, the results of the detection revealed that the mean plasma concentrations of $\mathrm{AC}$ and $\mathrm{MA}$ in HCD were higher than those in RCD, with a significant difference concerning $\mathrm{AUC}_{0-t}, C_{\max }$, and $t_{1 / 2 z}$, whereas there was no significant difference between those parameters in $\mathrm{HCP}$ and $\mathrm{HCD}$, implying that the influence of decocting on $\mathrm{RC}$ was greater than that on HC.

\section{Discussion}

In pharmacokinetics, after a medicine is administrated, the parameters $\mathrm{AUC}_{0-t}, C_{\max }$, and $T_{\max }$ are used for evaluating the level and rate of its absorption, and the parameter $t_{1 / 2 z}$ is used for evaluating the rate of its elimination. Small values of $\mathrm{AUC}_{0-t}$ and $C_{\max }$ mean a low level of its absorption and a small value of $C_{\max }$ and a big value of $T_{\max }$ mean a low rate of its absorption, whereas a small value of $t_{1 / 2 z}$ means a high rate of its elimination [13]. In our present study, the pharmacokinetic parameters of the 6 alkaloids in RCP, HCP, RCD, and $\mathrm{HCD}$ were all detected and compared in order to find the detoxification mechanism of HC.

By analysis of the results of the content detection of the 6 alkaloids in RCP and HCP (Table 1), it was found that the 
TABLe 7: Pharmacokinetic parameters of the 6 alkaloids in plasma $(n=6)$.

\begin{tabular}{|c|c|c|c|c|c|}
\hline Alkaloids & Groups & $\mathrm{AUC}_{0-t}(\mathrm{ng} / \mathrm{h} / \mathrm{mL})$ & $t_{1 / 2 z}(\mathrm{~h})$ & $T_{\max }(\mathrm{h})$ & $C_{\max }(\mathrm{ng} / \mathrm{mL})$ \\
\hline \multirow{4}{*}{$\mathrm{AC}$} & $\mathrm{RCP}$ & $10.31 \pm 3.56$ & $2.29 \pm 0.70$ & $0.50 \pm 0.00$ & $7.23 \pm 3.24$ \\
\hline & $\mathrm{HCP}$ & $4.96 \pm 1.72^{* \# \#}$ & $2.95 \pm 1.89^{\# \#}$ & $2.38 \pm 0.69^{* *}$ & $2.31 \pm 1.16^{* * \#}$ \\
\hline & $\mathrm{RCD}$ & $1.75 \pm 0.04^{* *}$ & $0.88 \pm 0.00^{* *}$ & $3.00 \pm 0.00^{* *}$ & $0.83 \pm 0.19^{* *}$ \\
\hline & $\mathrm{HCD}$ & $3.88 \pm 0.23^{* \# \#}$ & $2.73 \pm 1.19^{\# \#}$ & $2.50 \pm 0.71^{* *}$ & $1.60 \pm 0.35^{* * \#}$ \\
\hline \multirow{4}{*}{ MA } & $\mathrm{RCP}$ & $77.12 \pm 1.73$ & $0.90 \pm 0.06$ & $0.50 \pm 0.00$ & $37.03 \pm 1.35$ \\
\hline & $\mathrm{HCP}$ & $46.90 \pm 16.99^{* \# \#}$ & $2.78 \pm 1.59^{* \# \#}$ & $2.17 \pm 0.68^{* *}$ & $19.83 \pm 9.03^{* * \#}$ \\
\hline & $\mathrm{RCD}$ & $10.23 \pm 3.22^{* *}$ & $0.30 \pm 0.03^{* *}$ & $2.25 \pm 0.47^{* *}$ & $5.89 \pm 2.22^{* *}$ \\
\hline & $\mathrm{HCD}$ & $61.86 \pm 3.92^{\# \#}$ & $2.56 \pm 1.94^{* \# \#}$ & $1.33 \pm 0.44$ & $25.88 \pm 4.30^{* * \# \#}$ \\
\hline \multirow{4}{*}{ HA } & $\mathrm{RCP}$ & $25.88 \pm 1.90$ & $1.61 \pm 0.66$ & $0.83 \pm 0.26$ & $16.07 \pm 4.54$ \\
\hline & $\mathrm{HCP}$ & $22.43 \pm 9.68$ & $3.37 \pm 1.49^{* \# \#}$ & $2.00 \pm 0.89^{* \#}$ & $10.58 \pm 6.86$ \\
\hline & $\mathrm{RCD}$ & $27.15 \pm 4.43$ & $0.88 \pm 0.00^{*}$ & $0.5 \pm 0.00$ & $14.75 \pm 5.80$ \\
\hline & $\mathrm{HCD}$ & $26.23 \pm 6.73$ & $4.45 \pm 1.13^{* * \# \#}$ & $1.33 \pm 0.44$ & $10.81 \pm 1.46$ \\
\hline \multirow{4}{*}{ BAC } & $\mathrm{RCP}$ & $6.74 \pm 0.68$ & $1.12 \pm 0.18$ & $0.5 \pm 0.00$ & $4.44 \pm 0.72$ \\
\hline & $\mathrm{HCP}$ & $2.97 \pm 0.60^{* *}$ & $0.58 \pm 0.17$ & $0.83 \pm 0.29$ & $1.79 \pm 0.25^{* *}$ \\
\hline & $\mathrm{RCD}$ & - & - & - & - \\
\hline & HCD & - & - & - & - \\
\hline \multirow{4}{*}{ BMA } & $\mathrm{RCP}$ & $42.34 \pm 8.49$ & $0.97 \pm 0.2$ & $0.83 \pm 0.52$ & $27.86 \pm 6.96$ \\
\hline & $\mathrm{HCP}$ & $17.28 \pm 5.36^{* * \#}$ & $4.68 \pm 0.96^{* *}$ & $2.00 \pm 0.65^{*}$ & $7.67 \pm 0.89^{* *}$ \\
\hline & $\mathrm{RCD}$ & $29.58 \pm 4.91$ & $6.65 \pm 1.98^{* *}$ & $1.5 \pm 0.00$ & $10.25 \pm 0.94^{*}$ \\
\hline & $\mathrm{HCD}$ & $15.29 \pm 4.61^{* * \#}$ & $1.8 \pm 0.00^{* \# \#}$ & $2.67 \pm 0.53^{*}$ & $6.38 \pm 2.34^{* *}$ \\
\hline \multirow{4}{*}{ BHA } & $\mathrm{RCP}$ & $16.76 \pm 2.12$ & $0.85 \pm 0.30$ & $1.50 \pm 0.13$ & $9.93 \pm 1.83$ \\
\hline & $\mathrm{HCP}$ & $3.54 \pm 0.55^{* *}$ & $1.33 \pm 0.58$ & $1.00 \pm 0.55$ & $3.16 \pm 0.79^{* *}$ \\
\hline & $\mathrm{RCD}$ & - & - & - & - \\
\hline & $\mathrm{HCD}$ & - & - & - & - \\
\hline
\end{tabular}

Mean \pm S.D., $n=6 .{ }^{*} p<0.05$ and ${ }^{* *} p<0.01$ vs. RCP group; ${ }^{*} p<0.05$ and ${ }^{\# \#} p<0.01$ vs. RCD group.

difference between the contents of the diester and those of the monoester diterpenoid alkaloids was little. Nevertheless, the results of the pharmacokinetic detection (Table 7) showed that the $\mathrm{AUC}_{0-t}$ and $C_{\max }$ of both the diester and monoester diterpenoid alkaloids were lower and $T_{\max }$ was higher in $\mathrm{HCP}$, compared with RCP, suggesting that the Hezi-processing method could cause a decrease in the level and rate of absorption of all kinds of alkaloids in Caowu which may be the mechanism of detoxification of the Hezi-processing method. The results of the pharmacokinetic detection also showed that the $t_{1 / 2 z}$ of most alkaloids in HCP was higher than that in RCP, implying that the Hezi-processing method for Caowu could also decrease the rate of elimination of the alkaloids in HCP and prolong their duration of action. In TMM, HC was frequently used in the dosage forms of pills and powder usually in a small dose. The results of our present study could verify that when $\mathrm{HC}$ was administrated in the dosage form of powder or pills, it would be much safer and exert a desirable effect in a relatively small dose.

By comparing the results of the content detection of the 6 alkaloids in RCD and RCP (Table 1), it was found that the contents of the diester diterpenoid alkaloids markedly decreased and the contents of the monoester diterpenoid alkaloids markedly increased in RCD. The results of the pharmacokinetic detection after being administrated with RCD and RCP (Table 7) showed that in RCD, the values of $\mathrm{AUC}_{0-t}$ and $C_{\max }$ of the diester diterpenoid alkaloids decreased, the value of $T_{\max }$ increased, and some monoester diterpenoid alkaloids (such as BAC and BHA) were unable to be detected, suggesting that these monoester diterpenoid alkaloids may have undergone a further hydrolysis. The results implied that decocting could accelerate hydrolysis of all the alkaloids in RC and thereby decrease the concentrations of these alkaloids in the plasma, which contributed to the detoxification of RC. It could also be seen, from the results of pharmacokinetic detection (Table 7 ), that the value of $t_{1 / 2 z}$ significantly decreased in RCD, suggesting that decocting could increase the rate of elimination of diester diterpenoid alkaloids in $\mathrm{RC}$, which also contributed to the detoxification of RC. The above-mentioned results of our present study may demonstrate the rationality of the various decocting methods used 
for processing $\mathrm{RC}$ and preparing Caowu-containing herb decoctions in TCM.

The results of the analysis of the contents of alkaloids in various Caowu samples (Table 1) showed that the contents of diester diterpenoid alkaloids in HCD were lower than those in HCP, and the contents of monoester diterpenoid alkaloids in HCD were higher than those in HCP, which was similar to the change in the contents of the various alkaloids in RCD and RCP; nothing but the level of the change was smaller, suggesting that there was a restriction of the hydrolysis of the alkaloids in Caowu during the processing of $\mathrm{HC}$ [14]. The results of the detection of the pharmacokinetic parameters (Table 7 ) showed that there was no marked difference between the values of the pharmacokinetic parameters of the diester diterpenoid alkaloids in HCD and HCP. However, compared with those in RCP, the values of AU $\mathrm{C}_{0-t}$ and $C_{\max }$ in HCP and HCD were decreased while $T_{\max }$ and $t_{1 / 2 z}$ were increased, suggesting that in HCP and HCD, the level and rate of absorption of the diester diterpenoid alkaloids were reduced and the process of elimination of these alkaloids was prolonged [15]. It had to be pointed out that the decrease of the value of $\mathrm{AUC}_{0-t}$ of the diester diterpenoid alkaloids in HCD may be due to the low concentration of the alkaloids in the HCD sample, whereas that in HCP may be due to the restriction of the absorption of the diester diterpenoid alkaloids caused by Hezi. The diester diterpenoid alkaloids in HCP could be gradually transformed into monoester diterpenoid alkaloids during a slow hydrolysis in vivo, causing the contents of the monoester diterpenoid alkaloids to increase to a detectable level. However, the free monoester diterpenoid alkaloids in HCD may be rapidly hydrolyzed in vivo, and no more monoester diterpenoid alkaloids could be supplemented by hydrolysis of the diester diterpenoid alkaloids because their contents were very low in the sample, which could explain why no BAC and BHA could be detected in HCD.

\section{Conclusion}

From the above-mentioned results of our present study, it can be concluded that the alkaloids in RCP can be absorbed at a high level and a high rate, suggesting that RCP is clinically unsafe and $\mathrm{RC}$ is unsuitable for being used in the dosage form of powder; that the alkaloids in HCP can be absorbed at a lower level and eliminated at a lower rate, suggesting that HCP is clinically safe and HC is suitable for being used in the dosage form of powder in a smaller dose; and that decocting can make the alkaloids in $\mathrm{RC}$ rapidly hydrolyzed and thereby greatly decrease the level of their plasma concentration and the intensity of toxicity of RC. The results of our present study can also demonstrate the rationality of Caowu being used mostly in the dosage forms of pills and powder in TMM and mostly in the dosage form of decoction in TCM.

\section{Data Availability}

The data used to support the findings of this study are available from the first author (zmr6822@163.com) upon request.

\section{Conflicts of Interest}

All authors declare that there are no conflicts of interest related to this article.

\section{Acknowledgments}

This study was financially supported by the National Natural Science Foundation of China (No. 81774004) and Beijing Municipal Natural Science Foundation (No. 7182093).

\section{References}

[1] Chinese Pharmacopoeia Commission, Chinese Pharmacopoeia, China Medical Science and Technology Press, 2015.

[2] Q. Li, S. D. Sun, M. Y. Wang, C. F. Li, D. Yuan, and H. Z. Fu, "Chemical constituents and analgesic activity of Aconitum kusnezoffii Reichb," Journal of Chinese Pharmaceutical Sciences, vol. 27, no. 12, pp. 855-863, 2018.

[3] A. P. Liu and Z. F. Yu, "Effects of Radix Aconiti Kusnezoffii on compound action potential of sciatic nerve of toad," Lishizhen Medicine and Materia Medica Research, vol. 19, no. 5, pp. 1109-1110, 2008.

[4] C. C. Du, Experimental Study and Mechanism of Aconitine Anti-Rheumatoid Arthritis, Guizhou Medical University, 2019.

[5] S. Liu, Study on the Influence of Processing Adjuvants on the Chemical Compositions of Aconiti Kusnezoffii Radix Processed with Chebulae Fructus and the Principle of Reducing Toxicity, Beijing University of Chinese Medicine, 2017.

[6] Y. Xin, S. M. Wang, W. G. Zhang, J. Lv, Y. Zhang, and Z. Zhang, "Chemical detoxification of fruit of medicine Terminalia on root of kusnezoffii monkshood by mass spectrometry," Chinese Journal of Experimental Traditional Medical Formulae, vol. 20, no. 23, pp. 51-56, 2014.

[7] H. M. Chen, "A comparison between the two total aconitine content in Aconitum Kusnezo ffii (Caowu) got by Mongolia medicine preparing method and by traditional Chinese drug preparing method and processing times influence to the content," Journal of Inner Mongolia University for Nationalities, vol. 21, no. 5, pp. 541-542, 2006.

[8] S. Liu, X. Y. Liu, Y. Li et al., "Experimental study on acute toxicity of the Aconiti Kusnezoffii Radix and its processed products," Chinese Journal of Pharmacovigilance, vol. 12, no. 9, pp. 513-516, 2015.

[9] Y. Liang, R. Xing, J. L. Liu et al., "Advances of novel technologies and theories in pharmacokinetic research," Journal of China Pharmaceutical University, vol. 45, no. 6, pp. 607-616, 2014.

[10] J. Liu, Q. Li, R. Liu, Y. Yin, X. Chen, and K. Bi, "Enrichment and purification of six Aconitum alkaloids from Aconiti kusnezoffii radix by macroporous resins and quantification by HPLC-MS," Journal of Chromatography B, vol. 960, pp. 174181, 2014.

[11] J. Singhuber, M. Zhu, S. Prinz, and B. Kopp, "Aconitum in traditional Chinese medicine-a valuable drug or an unpredictable risk?," Journal of Ethnopharmacology, vol. 126, no. 1, pp. 18-30, 2009.

[12] Y. J. Shen, Chinese Medical Herbology and Pharmacology, People's Medical Publishing House, Beijing, China, 2011.

[13] Q. S. Zheng and R. Y. Sun, "Functions of the DAS software for pharmacological calculation," Chinese Journal of Clinical 
Pharmacology and Therapeutics, vol. 7, no. 6, pp. 562-564, 2002.

[14] S. Liu, F. Li, Y. F. Hou et al., "Influence of tannins from Chebulae Fructus on Aconitum alkaloids of Aconitum Kusnezoffii processed with Chebulae Fructus-principal of Aconitum processed with Chebulae Fructus I," Chinese Journal of Experimental Traditional Medical Formulae, vol. 19, no. 5, pp. 158-160, 2013.

[15] X. M. An, W. Sheng, Y. Lin, and W. Wang, "Comparative study on the efficacy of different processing methods of Aconiti Kusnezoffii Radix," Nei Mongol Journal of Traditional Chinese Medicine, vol. 33, no. 7, pp. 89-91, 2014. 